\title{
Lattice dynamics of epitaxial strain-free interfaces
}

\author{
J. Kalt, ${ }^{1,2}$ M. Sternik, ${ }^{3}$ I. Sergueev, ${ }^{4}$ J. Herfort,${ }^{5}$ B. Jenichen, ${ }^{5}$ H.-C. Wille, ${ }^{4}$ O. Sikora, ${ }^{3}$ P. Piekarz, ${ }^{3}$ \\ K. Parlinski, ${ }^{3}$ T. Baumbach, ${ }^{1,2}$ and S. Stankov ${ }^{1,2, *}$ \\ ${ }^{1}$ Laboratory for Applications of Synchrotron Radiation, Karlsruhe Institute of Technology, D-76131 Karlsruhe, Germany \\ ${ }^{2}$ Institute for Photon Science and Synchrotron Radiation, Karlsruhe Institute of Technology, D-76344 Eggenstein-Leopoldshafen, Germany \\ ${ }^{3}$ Institute of Nuclear Physics, Polish Academy of Sciences, PL-31342 Kraków, Poland \\ ${ }^{4}$ Deutsches Elektronen-Synchrotron, D-22607 Hamburg, Germany \\ ${ }^{5}$ Paul-Drude-Institut für Festkörperelektronik, D-10117 Berlin, Germany
}

(Received 12 March 2018; published 24 September 2018)

\begin{abstract}
We report a systematic lattice dynamics study of the technologically important $\mathrm{Fe}_{3} \mathrm{Si} / \mathrm{GaAs}$ heterostructure for $\mathrm{Fe}_{3} \mathrm{Si}$ layer thicknesses of 3, 6, 8, and 36 monolayers. The Fe-partial phonon density of states obtained by nuclear inelastic scattering exhibits up to a twofold enhancement of the low-energy phonon states compared to the bulk material for layer thicknesses of 8 monolayers and below. First-principles calculations explain the observed effect by interface-specific phonon states originating from the significantly reduced atomic force constants and allow for achieving a comprehensive understanding of the lattice dynamics of epitaxial strain-free interfaces.
\end{abstract}

DOI: 10.1103/PhysRevB.98.121409

The contact area between two dissimilar materials is very often a source of new physical phenomena that govern many modern technologies such as opto- and nanoelectronics [1], photovoltaics [2], and magnetic recording [3,4], which influence remarkably our everyday life. Unlike the electronic and magnetic properties that have extensively been investigated and well understood, the propagation of thermal lattice excitations (phonons) along and across epitaxial interfaces remains poorly understood despite decades of research. The importance of the lattice dynamics of lattice-matched interfaces arises from the fact that it is intimately related to physical phenomena such as nanoscale thermal transport [5] and phonon filtering [6], which are fundamentally important for waste heat harvesting [7,8], thermal management in nanoelectronics $[9,10]$, and are the basis for the development of new logic devices operating at $\mathrm{THz}$ frequencies [11-13]. Furthermore, via electron-phonon [14] and magnon-phonon [15] interactions the lattice dynamics influences many physical properties, thereby offering the opportunity for their manipulation [16].

Interface-specific vibrational phenomena such as phonon folding [17-19], localized vibrational modes [20], coherent phonon heat conduction [21], crossover from incoherent to coherent phonon scattering [22], and enhanced thermal conductivity $[23,24]$ have experimentally been observed and theoretically studied. Yet, a comprehensive understanding of the lattice dynamics at the atomistic level even in the simplest case of a perfectly matched interface between dissimilar materials, leading, for example, to the Kapitza resistance [25,26], is still missing. Recent progress in theory made calculations of the phonon density of states (PDOS) of interface atoms possible and allowed for the identification of the vibrational modes responsible for heat transfer across the interface [27-29].

\footnotetext{
*svetoslav.stankov@kit.edu
}

The experimental observation of interface PDOS, however, remained a challenge until now.

Here, we present a combined experimental and theoretical lattice dynamics study of the strain-free $\mathrm{Fe}_{3} \mathrm{Si} / \mathrm{GaAs}$ heterostructure [30], which is a promising candidate for applications in magnetic and spintronic devices [31-33]. The Fe-partial PDOS of thin $\mathrm{Fe}_{3} \mathrm{Si}$ layers, obtained from nuclear inelastic scattering, reveals drastic deviations from the bulk behavior. First-principles calculations demonstrate that the observed anomalies originate from interface-specific phonon states induced by significantly reduced atomic force constants and allow for their comprehensive understanding at the microscopic level.

$\mathrm{Fe}_{3} \mathrm{Si}(001) / \mathrm{GaAs}(001)$ heterostructures with $\mathrm{Fe}_{3} \mathrm{Si}$ layer thicknesses of $36,8,6$, and $3 \mathrm{ML}$ (one monolayer ML is $0.28 \mathrm{~nm}$ ) [34] referred to as S1, S2, S3, and S4, respectively, were grown via molecular beam epitaxy [35,36] using iron enriched to $96 \%$ in ${ }^{57} \mathrm{Fe}$. The samples were covered with $4 \mathrm{~nm}$ of amorphous Ge to prevent oxidation and to eliminate surface vibrational modes. Electron and x-ray diffraction, and transmission electron microscopy confirmed that in all samples epitaxial, strain-free, two-dimensional structures are formed [37]. $\mathrm{Fe}_{3} \mathrm{Si}[100]$-projected, Fe-partial PDOS was obtained [38] by nuclear inelastic scattering $[39,40]$ performed at the Dynamics Beamline P01 [41] of PETRA III with an energy resolution of $0.9 \mathrm{meV}$ at grazing-incidence geometry.

Figure 1(a) shows the Fe-partial PDOS of samples S1-S4. The spectrum of $\mathrm{S} 1$ exhibits distinct peaks at 17, 34, and 41.5 $\mathrm{meV}$ and overlapping modes between 21.5 and $28 \mathrm{meV}$. Upon reduction of the $\mathrm{Fe}_{3} \mathrm{Si}$ layer thickness all peaks become broadened and suppressed, with the effect being most pronounced in S4. In addition, the PDOS both at low and high energies, as well as around 20 and $37 \mathrm{meV}$, is gradually enhanced. The reduced PDOS (PDOS $/ E^{2}$ ), plotted in Fig. 1(b), illustrates that in all samples the low-energy part obeys the Debye law $g(E)=\alpha E^{2}$, where $\alpha$ quantifies the enhancement. The inset 


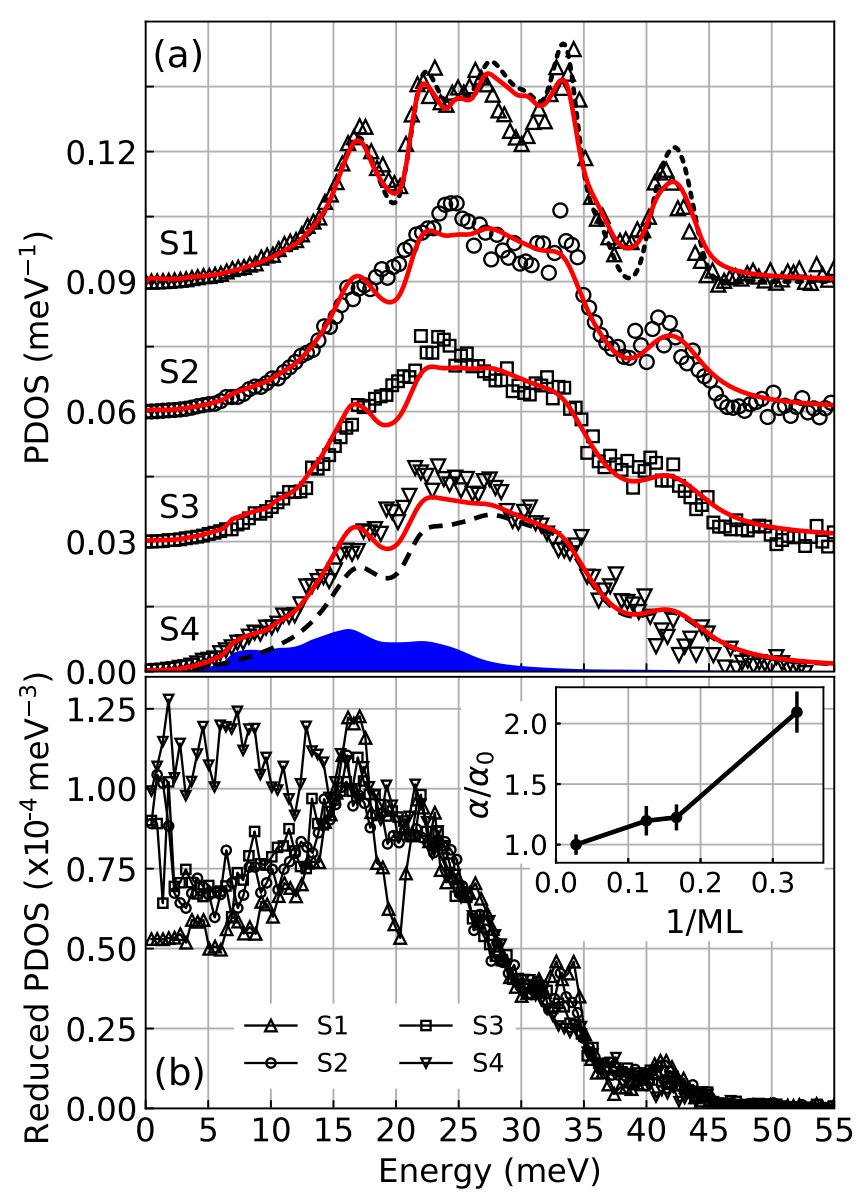

FIG. 1. (a) $\mathrm{Fe}_{3} \mathrm{Si}[100]$-projected, Fe-partial PDOS of the investigated samples. The spectra are upshifted by $0.03 \mathrm{meV}^{-1}$ for clarity. The dotted line marks the bulk PDOS convoluted with a Gauss function (full width at half maximum FWHM $=1.9 \mathrm{meV}$ ), the solid/red lines represent the function $g_{\text {theor }}(E)$, while the blue area and dashed line stand for the $a b$ initio calculated interface $g_{\text {if }}\left(E, Q_{\text {if }}\right)$ and bulk $g_{\text {bulk }}\left(E, Q_{\text {bulk }}\right)$ PDOS, respectively (see text). (b) The reduced PDOS (PDOS $/ E^{2}$ ) of the studied samples. The inset shows the normalized low-energy enhancement as a function of the inverse number of monolayers.

of Fig. 1(b) plots the value of $\alpha$ in samples S1-S4 normalized to that in $\mathrm{S} 1\left(\alpha_{0}\right)$. It demonstrates that the number of lowenergy states is twofold augmented in S4 compared to S1. Similar behavior, namely, a threefold and almost fourfold increase of the number of phonon states below $\approx 10 \mathrm{meV}$, was reported for the native $\mathrm{Fe}(110)$ surface [42] and ultrathin $\mathrm{Fe}(110)$ films deposited on $\mathrm{W}(110)$ [43]. In these systems the phenomenon was attributed to surface-specific modes and tensile epitaxial strain. In the Ge-covered and lattice-matched $\mathrm{Fe}_{3} \mathrm{Si} / \mathrm{GaAs}$ heterostructure investigated here, however, these sources of vibrational anomalies are ruled out, implying that the observed low-energy enhancement originates from interface-specific PDOS, as demonstrated below.

The experimental PDOS is composed of vibrations of Fe atoms located at different layers within the epitaxial system. In order to elucidate the contributions of atoms occupying the interface and internal layers, first-principles theory was applied using the direct method [44] implemented in the

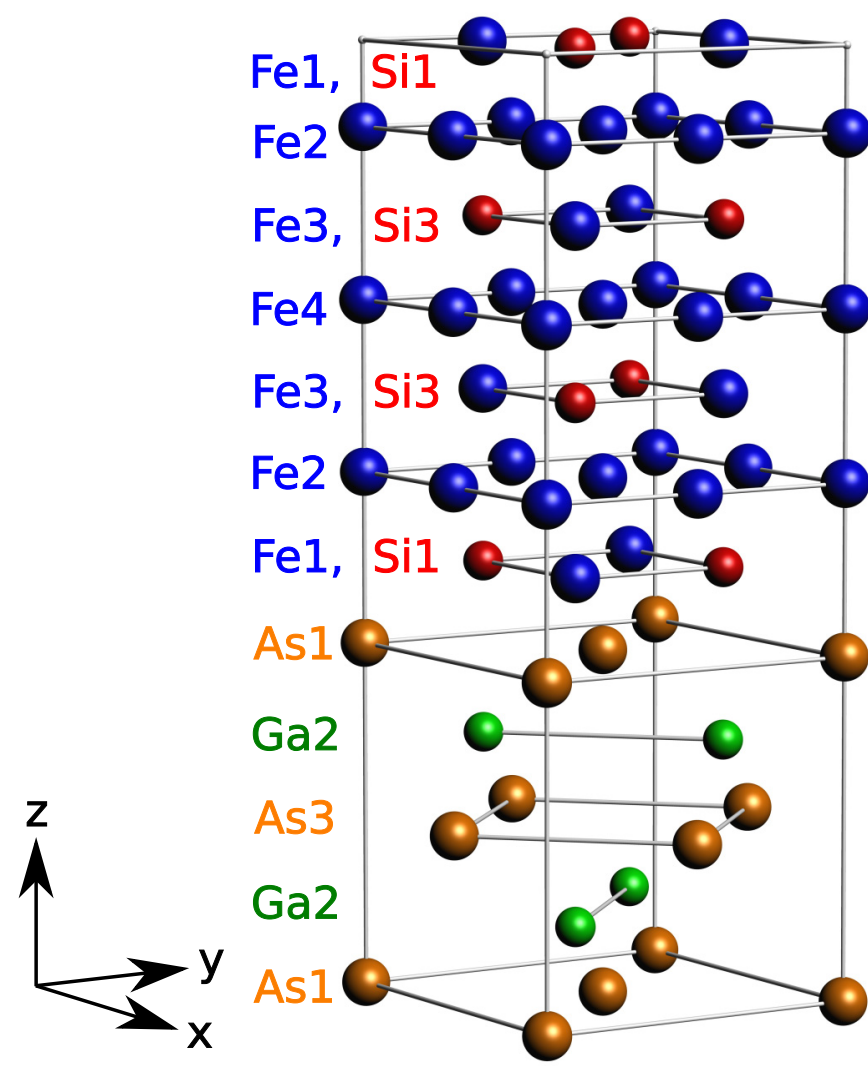

FIG. 2. The atomic configuration of the $\mathrm{Fe}_{3} \mathrm{Si}(001) / \mathrm{GaAs}(001)$ multilayer used to calculate the interface lattice dynamics.

PHONON code [45]. System optimization and calculations of the Hellmann-Feynman forces were performed using the spin-polarized density functional theory with the generalizedgradient approximation [46] and the projector augmentedwave method [47] implemented in the VASP software [48]. The phonon calculations for bulk crystal were performed in a $2 \times$ $2 \times 2$ supercell containing 128 atoms with periodic boundary conditions. The obtained phonon frequencies [37] are in very good agreement with the results from inelastic neutron scattering experiment [49] and earlier ab initio calculations [50,51].

The $\mathrm{Fe}_{3} \mathrm{Si} / \mathrm{GaAs}$ interface was modeled by a multilayer consisting of 12 atomic layers (Fig. 2) using a $\sqrt{2} a \times \sqrt{2} a \times$ $c$ supercell with 76 atoms and periodic boundary conditions. Four types of atomic arrangements at the $\mathrm{Fe}_{3} \mathrm{Si} / \mathrm{GaAs}$ interface [52] were examined: two configurations exhibiting mixed Fe-Si layers and two configurations characterized by pure Fe layers directly adjacent to the As-terminated GaAs(001) surface. A comparison of the experimental data with the theoretical results (see below) showed that the variant presented in Fig. 2, with a mixed Fe-Si interface layer, leads to the best agreement, hence in the following only this interface atomic configuration is considered.

Figure 3 summarizes the ab initio calculated layer-specific Fe- and Si-partial PDOS with polarizations along $(x y)$ and across $(z)$ the interface (solid lines) compared with the corresponding spectra in bulk $\mathrm{Fe}_{3} \mathrm{Si}$ (gray area). Figures 3(a) and 3(b) demonstrate that the Fe atoms of the first interface layer (Fe1 in Fig. 2) exhibit the largest vibrational anomalies in both polarizations. Along the interface ( $x y$ projection), new 


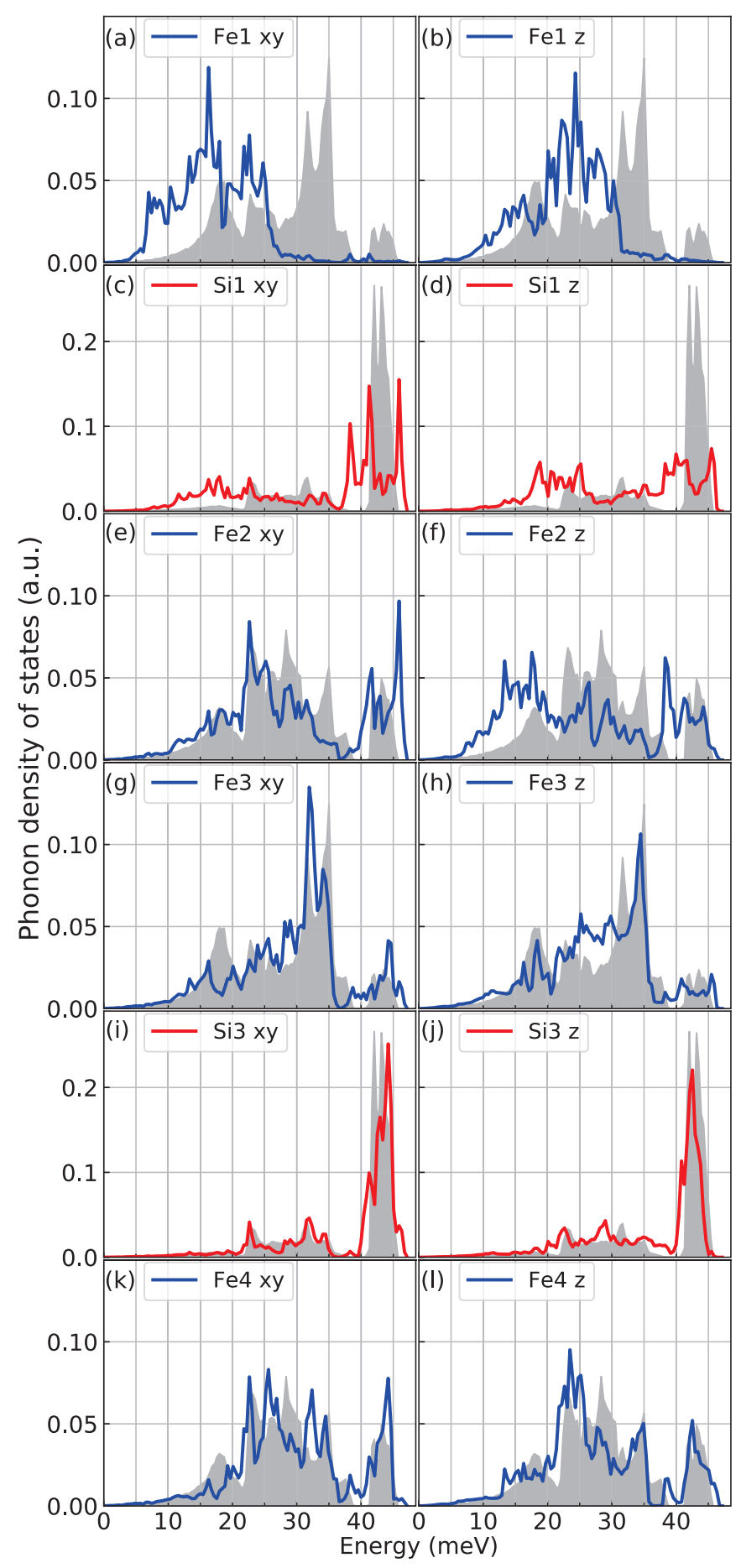

FIG. 3. The $a b$ initio calculated layer-specific Fe- and Si-partial PDOS projected along [(a), (c), (e), (g), (i), (k)] and across [(b), (d), (f), (h), (j), (l)] the interface (solid lines) compared with the corresponding spectra (gray area) in bulk $\mathrm{Fe}_{3} \mathrm{Si}$. The color and layer numbers correspond to those in Fig. 2.

vibrational modes appear between 6 and $18 \mathrm{meV}$ and between 20 and $25 \mathrm{meV}$ [Fig. 3(a)], leading to a remarkable enhancement of the low-energy states compared to the bulk crystal. Although less pronounced, augmented low-energy states are also present in the $z$-projected PDOS [Fig. 3(b)] along with a new set of modes between 20 and $30 \mathrm{meV}$. The high-energy

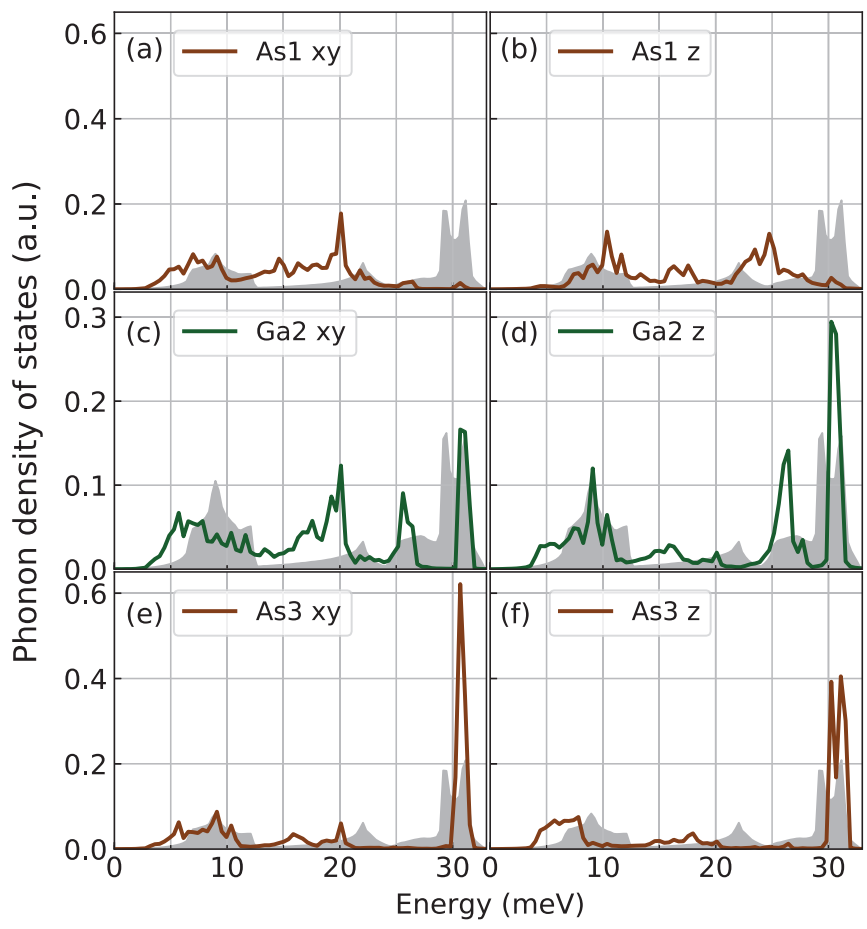

FIG. 4. The ab initio calculated layer-specific As- and Ga-partial PDOS projected along [(a), (c), (e)] and across [(b), (d), (f)] the interface (solid lines) compared with the corresponding PDOS (gray area) in bulk GaAs. The color and layer numbers correspond to those in Fig. 2.

cutoff is reduced to $27 \mathrm{meV}$ for the $x y$ and $32 \mathrm{meV}$ for the $z$ polarization, indicating a drastic lattice softening. In the Sil layer, the number of states below $23 \mathrm{meV}$ increases and the peaks around $43 \mathrm{meV}$ are suppressed and split into three modes in both polarizations [Figs. 3(c) and 3(d)]. In the $x y$-projected PDOS of the Fe2 layer the peaks at 28 and $35 \mathrm{meV}$ are suppressed [Fig. 3(e)]. Figure 3(f) demonstrates that although the high-energy cutoff corresponds to that in bulk $\mathrm{Fe}_{3} \mathrm{Si}$, notable deviations are still present across the entire spectrum of the $z$-projected PDOS. The PDOS of the iron atoms in the $\mathrm{Fe} 3$ layer deviates from the bulk at $18.5 \mathrm{meV}$ in $x y$ polarization [Fig. $3(\mathrm{~g})$ ] and between 23 and $32 \mathrm{meV}$ in $z$ polarization [Fig. 3(h)]. The high-energy vibrations of the $\mathrm{Si}$ atoms in this layer ( $\mathrm{Si} 3)$ are affected in both directions [Figs. 3(i) and 3(j)]. In the PDOS of the fourth pure iron layer Fe4 still minor deviations from the bulk spectrum are observed for both polarizations [Figs. 3(k) and 3(1)].

The broken translational symmetry at the $\mathrm{Fe}_{3} \mathrm{Si} / \mathrm{GaAs}$ interface remarkably influences also the vibrations of the GaAs layers. Theoretical results for the PDOS of the Ga and As layers are presented in Fig. 4. Significant deviations from the corresponding bulk spectra are observed in the PDOS of the first interface layer As1 (Fig. 2). New vibrational modes emerge in both polarizations, while the peaks around $30 \mathrm{meV}$ are fully suppressed [Figs. 4(a) and 4(b)]. Although the peak at $32 \mathrm{meV}$ reappears in the PDOS of the $\mathrm{Ga} 2$ atoms forming the second layer, it still deviates from the bulk spectrum [Figs. 4(c) and 4(d)]. The PDOS of the third layer (As3) also 
exhibits notable deviations from bulk features along the entire energy range [Figs. 4(e) and 4(f)].

The modifications of the PDOS of the interface and adjacent layers originate from the drastically reduced force constants (see Table I) due to the altered surroundings of the interface atoms compared to the corresponding layers in bulk material. The anomalies in the investigated heterostructure extend up to the fourth atomic layer [see Figs. 3(k) and 3(1)]. Similar effects are observed for the $\mathrm{Ge} / \mathrm{Fe}_{3} \mathrm{Si}$ interface [37], indicating that most likely this is a general trend for metal/semiconductor heterostructures.

The applied grazing-incidence scattering geometry implies that the in-plane projected PDOS is derived from the experiment [53]. The sharp peaks present in the theoretically obtained PDOS (Fig. 3) are gradually broadened (reduced phonon lifetime) and suppressed as the $\mathrm{Fe}_{3} \mathrm{Si}$ layer thickness is reduced [Fig. 1(a)]. This effect cannot be explained solely by broadening due to the experimental resolution. For instance, the dotted line in Fig. 1(a) denotes a fit of the PDOS of S1 with the bulk $a b$ initio calculated PDOS convoluted with Gaussian function with FWHM $=1.9 \mathrm{meV}$, which is by a factor of 2 higher than the experimental resolution $(0.9 \mathrm{meV})$. The additional broadening of the peaks is a characteristic feature of the PDOS of nanoscale materials that most likely originates from anharmonic interatomic potentials experienced by atoms located at irregular sites [54]. In the investigated samples, this is the case for the Fe atoms adjacent to the amorphous Ge layer and atomic defects at the epitaxial $\mathrm{Fe}_{3} \mathrm{Si} / \mathrm{GaAs}$ interface. This effect can satisfactorily be described by the damped harmonic oscillator (DHO) function characterized with a quality factor $Q$ [55]. The DHO induces energy-dependent broadening of the spectral features: The stronger the effect, the lower is the $Q$ value. A comparison of the calculated Fe-partial PDOS convoluted with the DHO function with $Q=21$ and the PDOS of sample S1 confirms the remarkable agreement between experiment and theory for bulk $\mathrm{Fe}_{3} \mathrm{Si}$ crystal [Fig. 1(a)].

The second anomalous feature in the experimental PDOS of samples S2-S4 is the gradual enhancement of the lowenergy phonon states [Fig. 1(b)]. To model the observed anomalies we use the function

$$
g_{\text {theor }}(E)=A g_{\text {if }}\left(E, Q_{\text {if }}\right)+(1-A) g_{\text {bulk }}\left(E, Q_{\text {bulk }}\right),
$$

with $g_{\text {if }}\left(E, Q_{\text {if }}\right)$ and $g_{\text {bulk }}\left(E, Q_{\text {bulk }}\right)$ being the $a b$ initio calculated $x y$-projected PDOS of the first interface layer $(\mathrm{Fe} 1)$ and bulk $\mathrm{Fe}_{3} \mathrm{Si}$, respectively, convoluted with the DHO function with quality factors $Q_{\text {if }}$ and $Q_{\text {bulk }}$, while $A$ stands for the relative interface $(\mathrm{Fe} 1)$ atomic fraction. The experimental PDOS were fitted with Eq. (1) using the least-squares method with $Q_{\text {bulk }}, Q_{\text {if }}$, and $A$ being variable parameters. The results for S4 are $Q_{\text {bulk }}=7, Q_{\text {if }}=8$, and $A=0.15$. Figure 1(a) demonstrates that the model reproduces remarkably well the experimental data [56]. This result implies that the interfacespecific PDOS of the Fe1 layer [blue area in Fig. 1(a)] is responsible for the observed drastic enhancement of lowenergy states [57]. The minor deviation in the range 17-27 meV may arise from the additional modes induced by the $\mathrm{Fe}_{3} \mathrm{Si} / \mathrm{Ge}$ interface that is not included in the model [58].

For S3 the values are $Q_{\text {bulk }}=7, Q_{\text {if }}=15$, and $A=0.09$, whereas for S2 we obtained $Q_{\text {bulk }}=10, Q_{\text {if }}=15$, and
TABLE I. Experimental (Expt.) and theoretical (Theor.) values of the mean force constant $F(\mathrm{~N} / \mathrm{m})$, mean square displacement $\left\langle x^{2}\right\rangle\left(\AA^{2}\right)$, and vibrational entropy $S_{V}\left(k_{B} /\right.$ atom $)$ of the Fe atoms in samples S1-S4 and the $x y$ - and z-projected values for the Fe1 layer of the $\mathrm{Fe}_{3} \mathrm{Si} / \mathrm{GaAs}$ interface (Fig. 2).

\begin{tabular}{lllllllr}
\hline \hline & & S1/bulk & \multicolumn{1}{c}{ S2 } & \multicolumn{1}{c}{ S3 } & S4 & $x y$ & $z$ \\
\hline$F$ & Expt. & $175(3)$ & $173(3)$ & $172(4)$ & $159(5)$ & & \\
& Theor. & 176 & 170 & 167 & 161 & 74 & 121 \\
$\left\langle x^{2}\right\rangle$ & Expt. & $0.014(2)$ & $0.015(2)$ & $0.016(2)$ & $0.019(3)$ & & \\
& Theor. & 0.018 & 0.019 & 0.020 & 0.021 & 0.039 & 0.020 \\
$S_{V}$ & Expt. & $3.19(2)$ & $3.22(2)$ & $3.24(2)$ & $3.38(3)$ & & \\
& Theor. & 3.146 & 3.225 & 3.265 & 3.345 & 4.470 & 3.616 \\
\hline \hline
\end{tabular}

$A=0.06$. The electron microscopy study [37] showed that in S4 two-dimensional islands with heights between 6 and 8 atomic layers are formed, implying a $1 / 7(0.14)$ contribution of the first interface layer. In $\mathrm{S} 3$ and $\mathrm{S} 2$, the $\mathrm{Fe}_{3} \mathrm{Si}$ film consists of 12 and 16 atomic layers, respectively, indicating that these contributions are $1 / 12(0.08)$ for $\mathrm{S} 3$ and $1 / 16(0.06)$ for $\mathrm{S} 2$ from the total atomic fraction. These values are fully consistent with the relative interface atomic fractions $(A)$ obtained by the fitting procedure.

A comparison of the quality factors shows that $Q_{\text {bulk }}$ remains unchanged in S3 and S4, while its value increases in $\mathrm{S} 2$, whereas $Q_{\text {if }}$ is relatively low in $\mathrm{S} 4$ and increases in $\mathrm{S} 3$ and S2. This behavior is attributed to the fact that in $\mathrm{S} 3$ and S4 the value of $Q_{\text {bulk }}$ is strongly influenced by the $\mathrm{Ge} / \mathrm{Fe}_{3} \mathrm{Si}$ interface. Indeed, $\mathrm{Fe}_{3} \mathrm{Si}$ forms two-dimensional islands in $\mathrm{S} 4$ and two-dimensional islands on top of a thin layer in S3 [37]. In contrast, a continuous film is formed in S2 [37], implying a reduced $\mathrm{Fe}_{3} \mathrm{Si} / \mathrm{Ge}$ interface contribution. The smaller this area, the weaker are the disorder effects arising from the crystalline/amorphous interface, and the higher is the quality factor. At the $\mathrm{Fe}_{3} \mathrm{Si} / \mathrm{GaAs}$ interface, on the other hand, the GaAs substrate is completely covered in samples S2 and S3, whereas in $\mathrm{S} 4$ the periodicity is broken along the interface. In the latter, the amorphous Ge layer induces a certain degree of disorder at the island edges. This effect, along with the higher relative contribution of unavoidable atomic defects at the $\mathrm{Fe}_{3} \mathrm{Si} / \mathrm{GaAs}$ interface, lead to a reduction of $Q_{\text {if }}$ compared to $\mathrm{S} 2$ and $\mathrm{S} 3$.

The modified lattice dynamics at the interface impacts the thermoelastic properties calculated from PDOS [59], as demonstrated in Table I. The experimentally derived Fepartial values of the mean force constant $F$, mean square displacement $\left\langle x^{2}\right\rangle$, and vibrational entropy $S_{V}$ for S1-S4 are compared to the values obtained from $g_{\text {theor }}(E)$ and to the results for the Fe1 layer (Fig. 2) in $x y$ and $z$ projection. $F$ decreases by $9 \%$ in $\mathrm{S} 4$ compared to $\mathrm{S} 1$. The softening is most distinct along the first interface layer, reaching a reduction of $58 \%$, while across the interface it amounts to $31 \%$ from the bulk value. $\left\langle x^{2}\right\rangle$ increases by reducing the layer thickness reaching an enhancement of $36 \%$ in $\mathrm{S} 4$ compared to S1. At the interface, $\left\langle x^{2}\right\rangle$ increases by a factor of 2.2 in the $x y$ projection and by 1.1 in the $z$ projection. $S_{V}$ systematically increases by reducing the layer thickness, reaching $42 \%$ from the bulk value along the interface, whereas across the interface the effect is $15 \%$. The very good agreement between experiment 
and theory confirms that first-principles theory reliably explains the observed phenomena.

In summary, we performed a systematic lattice dynamics study of the strain-free $\mathrm{Fe}_{3} \mathrm{Si} / \mathrm{GaAs}$ heterostructure for $\mathrm{Fe}_{3} \mathrm{Si}$ layer thicknesses between 3 and 36 monolayers. The Fe-partial PDOS exhibits up to a twofold enhancement of the low-energy states compared to bulk material, as well as broadening and suppression of the peaks for layer thicknesses of 8 monolayers and below. First-principles calculations unveil that the observed anomalies at low energies originate from interface-specific phonon states. The broken translational symmetry at the interface drastically reduces the atomic force constants, with the effect being stronger along the interface. The broadening and suppression of the peaks are induced by the amorphous Ge capping layer and by defects at the $\mathrm{Fe}_{3} \mathrm{Si} / \mathrm{GaAs}$ interface. By departure from the interface, the vibrational anomalies vanish and bulk lattice dynamics sets in within several atomic layers.

The presented quantitative results pave the way towards phonon nanoengineering in two-dimensional systems that will have implications on designing efficient thermoelectric heterostructures and stimulate further progress in thermal management and nanophononics.

We thank H. P. Schönherr and C. Hermann for the support in the MBE laboratory at PDI. S.S. acknowledges the financial support by the Helmholtz Association (VH-NG-625) and BMBF (05K16VK4). P.P. acknowledges the financial support by Narodowe Centrum Nauki (NCN, National Science Centre) under Project No. 2017/25/B/ST3/02586.
[1] M. Osada and T. Sasaki, Adv. Mater. 24, 210 (2012).

[2] M. Graetzel, R. A. Janssen, D. B. Mitzi, and E. H. Sargent, Nature (London) 488, 304 (2012).

[3] M. N. Baibich, J. M. Broto, A. Fert, F. Nguyen Van Dau, F. Petroff, P. Etienne, G. Creuzet, A. Friederich, and J. Chazelas, Phys. Rev. Lett. 61, 2472 (1988).

[4] G. Binasch, P. Grünberg, F. Saurenbach, and W. Zinn, Phys. Rev. B 39, 4828 (1989).

[5] S. Volz, J. Ordonez-Miranda, A. Shchepetov, M. Prunnila, J. Ahopelto, T. Pezeril, G. Vaudel, V. Gusev, P. Ruello, and E. M. Weig, Eur. Phys. J. B 89, 15 (2016).

[6] V. Narayanamurti, H. L. Störmer, M. A. Chin, A. C. Gossard, and W. Wiegmann, Phys. Rev. Lett. 43, 2012 (1979).

[7] G. J. Snyder and E. S. Toberer, Nat. Mater. 7, 105 (2008).

[8] K. Biswas, J. He, I. D. Blum, C.-I. Wu, T. P. Hogan, D. N. Seidman, V. P. Dravid, and M. G. Kanatzidis, Nature (London) 489, 414 (2012).

[9] E. Pop, Nano Res. 3, 147 (2010).

[10] A. L. Moore and L. Shi, Mater. Today 17, 163 (2014).

[11] L. Wang and B. Li, Phys. Rev. Lett. 99, 177208 (2007).

[12] L. Wang and B. Li, Phys. Rev. Lett. 101, 267203 (2008).

[13] M. J. Martínez-Pérez, A. Fornieri, and F. Giazotto, Nat. Nanotechnol. 10, 303 (2015).

[14] N. Driza, S. Blanco-Canosa, M. Bakr, S. Soltan, M. Khalid, L. Mustafa, K. Kawashima, G. Christiani, H. Habermeier, and G. Khaliullin, Nat. Mater. 11, 675 (2012).

[15] R. Pradip, P. Piekarz, A. Bosak, D. G. Merkel, O. Waller, A. Seiler, A. I. Chumakov, R. Rüffer, A. M. Oleś, K. Parlinski, M. Krisch, T. Baumbach, and S. Stankov, Phys. Rev. Lett. 116, 185501 (2016).

[16] S. Calder, J. Lee, M. B. Stone, M. D. Lumsden, J. Lang, M. Feygenson, Z. Zhao, J.-Q. Yan, Y. Shi, and Y. Sun, Nat. Commun. 6, 89161 (2015).

[17] C. Colvard, R. Merlin, M. V. Klein, and A. C. Gossard, Phys. Rev. Lett. 45, 298 (1980).

[18] C. Colvard, T. A. Gant, M. V. Klein, R. Merlin, R. Fischer, H. Morkoc, and A. C. Gossard, Phys. Rev. B 31, 2080 (1985).

[19] A. K. Sood, J. Menendez, M. Cardona, and K. Ploog, Phys. Rev. Lett. 54, 2111 (1985).

[20] A. Seiler, P. Piekarz, S. Ibrahimkutty, D. G. Merkel, O. Waller, R. Pradip, A. I. Chumakov, R. Rüffer, T. Baumbach, K.
Parlinski, M. Fiederle, and S. Stankov, Phys. Rev. Lett. 117, 276101 (2016).

[21] M. N. Luckyanova, J. Garg, K. Esfarjani, A. Jandl, M. T. Bulsara, A. J. Schmidt, A. J. Minnich, S. Chen, M. S. Dresselhaus, and Z. Ren, Science 338, 936 (2012).

[22] J. Ravichandran, A. K. Yadav, R. Cheaito, P. B. Rossen, A. Soukiassian, S. Suresha, J. C. Duda, B. M. Foley, C.-H. Lee, and Y. Zhu, Nat. Mater. 13, 168 (2014).

[23] D. P. Schroeder, Z. Aksamija, A. Rath, P. M. Voyles, M. G. Lagally, and M. A. Eriksson, Phys. Rev. Lett. 115, 256101 (2015).

[24] W. Park, A. Sood, J. Park, M. Asheghi, R. Sinclair, and K. E. Goodson, Nanoscale Microscale Thermophys. Eng. 21, 134 (2017).

[25] P. Kapitza, J. Phys. (USSR) 4, 181 (1941).

[26] S. Pettersson and G. D. Mahan, Phys. Rev. B 42, 7386 (1990).

[27] Y. Chalopin and S. Volz, Appl. Phys. Lett. 103, 051602 (2013).

[28] K. Gordiz and A. Henry, New J. Phys. 17, 103002 (2015).

[29] K. Gordiz and A. Henry, J. Appl. Phys. 119, 015101 (2016).

[30] V. M. Kaganer, B. Jenichen, R. Shayduk, W. Braun, and H. Riechert, Phys. Rev. Lett. 102, 016103 (2009).

[31] S. Gaucher, B. Jenichen, J. Kalt, U. Jahn, A. Trampert, and J. Herfort, Appl. Phys. Lett. 110, 102103 (2017).

[32] M. Kawano, K. Santo, M. Ikawa, S. Yamada, T. Kanashima, and K. Hamaya, Appl. Phys. Lett. 109, 022406 (2016).

[33] A. Kawaharazuka, M. Ramsteiner, J. Herfort, H.-P. Schönherr, H. Kostial, and K. Ploog, Appl. Phys. Lett. 85, 3492 (2004).

[34] $\mathrm{In} \mathrm{Fe}_{3} \mathrm{Si}(001)$ one monolayer consists of two layers: one layer solely made out of $\mathrm{Fe}$ atoms and one layer made out of an equal number of $\mathrm{Fe}$ and $\mathrm{Si}$ atoms.

[35] B. Jenichen, J. Herfort, U. Jahn, A. Trampert, and H. Riechert, Thin Solid Films 556, 120 (2014).

[36] J. Herfort, H.-P. Schönherr, and B. Jenichen, J. Appl. Phys. 103, 07B506 (2008).

[37] See Supplemental Material at http://link.aps.org/supplemental/ 10.1103/PhysRevB.98.121409 for sample preparation and characterization and $a b$ initio calculations.

[38] V. G. Kohn and A. I. Chumakov, Hyperfine Interact. 125, 205 (2000).

[39] M. Seto, Y. Yoda, S. Kikuta, X. W. Zhang, and M. Ando, Phys. Rev. Lett. 74, 3828 (1995). 
[40] W. Sturhahn, T. S. Toellner, E. E. Alp, X. Zhang, M. Ando, Y. Yoda, S. Kikuta, M. Seto, C. W. Kimball, and B. Dabrowski, Phys. Rev. Lett. 74, 3832 (1995).

[41] H.-C. Wille, H. Franz, R. Röhlsberger, W. A. Caliebe, and F. U. Dill, J. Phys.: Conf. Ser. 217, 012008 (2010).

[42] T. Ślezak, J. Łażewski, S. Stankov, K. Parlinski, R. Reitinger, M. Rennhofer, R. Rüffer, B. Sepiol, M. Ślezak, N. Spiridis, M. Zajac, A. I. Chumakov, and J. Korecki, Phys. Rev. Lett. 99, 066103 (2007).

[43] S. Stankov, R. Röhlsberger, T. Ślezak, M. Sladecek, B. Sepiol, G. Vogl, A. I. Chumakov, R. Rüffer, N. Spiridis, J. Łażewski, K. Parlinski, and J. Korecki, Phys. Rev. Lett. 99, 185501 (2007).

[44] K. Parlinski, Z. Q. Li, and Y. Kawazoe, Phys. Rev. Lett. 78, 4063 (1997).

[45] K. Parlinski, Software PHONON, Cracow, 2011.

[46] J. P. Perdew, K. Burke, and M. Ernzerhof, Phys. Rev. Lett. 77, 3865 (1996).

[47] P. E. Blöchl, Phys. Rev. B 50, 17953 (1994).

[48] G. Kresse and J. Furthmüller, Comput. Mater. Sci. 6, 15 (1996).

[49] O. G. Randl, G. Vogl, W. Petry, B. Hennion, B. Sepiol, and K. Nembach, J. Phys.: Condens. Matter 7, 5983 (1995).

[50] S. Dennler and J. Hafner, Phys. Rev. B 73, 174303 (2006).

[51] Y. F. Liang, S. Shang, J. Wang, Y. Wang, F. Ye, J. Lin, G. Chen, and Z. Liu, Intermetallics 19, 1374 (2011).

[52] V. M. Kaganer, B. Jenichen, R. Shayduk, and W. Braun, Phys. Rev. B 77, 125325 (2008).
[53] A. I. Chumakov and W. Sturhahn, Hyperfine Interact. 123, 781 (1999).

[54] B. Fultz, Prog. Mater. Sci. 55, 247 (2010).

[55] B. Fåk and B. Dorner, Institute Laue Langevin Technical Report No. 92FA008S, 1992 (unpublished).

[56] In particular, the steplike increase of the experimental PDOS at $7 \mathrm{meV}$ [Fig. 1(a)], clearly visible in the reduced PDOS [Fig. 1(b)], originates from the peak at $7 \mathrm{meV}$ in the $x y$ projected PDOS of Fe1 [Fig. 3(a)].

[57] Although deviations from bulk behavior are observed even in the PDOS of the fourth iron layer Fe4 [see Fig. 3(k)], the best agreement with experiment is achieved when only the $x y$ projected PDOS of the first interface layer Fe1 [Fig. 3(a)] is considered. The PDOS of this layer exhibits the most pronounced vibrational anomalies, whereas the PDOS of the other layers are characterized with smaller deviations from bulk features that cannot be captured by the experiment.

[58] Ab initio calculations have also been performed for the $\mathrm{Ge} / \mathrm{Fe}_{3} \mathrm{Si}$ interface and are presented in the Supplemental Material [37]. With the approach used here it was only possible to calculate a crystalline $\mathrm{Ge} / \mathrm{Fe}_{3} \mathrm{Si}$ interface, which does not account for the effects introduced at the amorphous/crystalline interface present in the investigated samples. Therefore, all analyses were done by taking into account only the $\mathrm{Fe}_{3} \mathrm{Si} / \mathrm{GaAs}$ interface.

[59] M. Y. Hu, T. S. Toellner, N. Dauphas, E. E. Alp, and J. Zhao, Phys. Rev. B 87, 064301 (2013). 